\title{
The impact of ultrasonography on quality of cardiopulmonary resuscitation: a retrospective observational study
}

Wan-Ching Lien

National Taiwan University Hospital

Kah-Meng Chong

National Taiwan University Hospital

Herman Chih-Heng Chang

National Taiwan University Hospital

Su-Fen Cheng

National Taiwan University Hospital

Wei-Tien Chang ( $\nabla$ wtchang@ntu.edu.tw )

National Taiwan University Hospital

Matthew Huei-Ming

National Taiwan University Hospital

Wen-Jone Chen

National Taiwan University Hospital

\section{Research}

Keywords: ultrasonography (US), cardiopulmonary resuscitation (CPR), pulse check, continuous ultrasonography (continuous US), pause

Posted Date: December 23rd, 2019

DOI: https://doi.org/10.21203/rs.2.19577/v1

License: (a) (1) This work is licensed under a Creative Commons Attribution 4.0 International License. Read Full License 


\section{Abstract}

Background: This study aims to evaluate the effect of ultrasonography (US) on quality of cardiopulmonary resuscitation (CPR), and US-related pause duration of pulse-checks.

Methods: This retrospective observational study was conducted at the emergency department of National Taiwan University Hospital between April 2017 and May 2018. Video recordings for adult patients with non-traumatic cardiac arrest in designated resuscitation rooms were collected. The primary outcome was chest compression fraction (CCF) in the CPR with US group. The secondary outcome was pause duration of pulse checks with introduction of US or not. US-related pulse-checks were stratified into US during hands-off periods only (hands-off US), and US performing from hands-off to hands-on periods (continuous US).

Results: A total of 153 patients were enrolled. Continuous US was performed in 116 patients. CCF was similar (92\%) between the CPR with and without US groups. In the CPR with US group, pause duration was significantly longer in US-related pulse-checks than that without US $(9.3 \pm 8.0$ v.s. $7.3 \pm 4.7 s, p<0.0001)$. Notably, longer pause was noted in the hands-off US, as comparing with that in the continuous US (18.3 \pm 16.4 v.s. $7.7 \pm 3.5 \mathrm{~s}, \mathrm{p}<0.0001)$.

Conclusions: The introduction of US during CPR did not impact on CCF. Individual pause would not be lengthened if continuous US was performed while allowing chest compressions to be resumed. In addition, structured training, adequate facilities and manpower, and a timer reminding resumption of chest compressions would help sophisticated integration of US into CPR process.

\section{Background}

Ultrasonography (US) is a real-time, non-invasive, readily accessible diagnostic tool, frequently deployed in the emergency departments (EDs). It has been increasingly used in certain critical scenarios such as shock, dyspnea and cardiac arrest[1-7] to search for possible reversible causes and guide clinical management. Current guidelines for advanced life support (ALS) suggest that US can be an integral part of resuscitative medicine[8].

Chest compressions at a rate of 100-120 per minute, and minimizing interruptions to achieve chest compression fraction (CCF) above $80 \%$, contribute to high-quality of cardiopulmonary resuscitation (CPR) [9]. Interruption of chest compressions impairs coronary and cerebral perfusion pressure, and compromises the outcomes of CPR $[10,11]$. CCF is an indicator of quality that higher CCF is associated with a higher rate of return of spontaneous circulation (ROSC) $[12,13]$.

Current guidelines recommend interruptions in CPR of no more than $10 \mathrm{~s}[14,15]$. It has been debated whether employment of US during CPR lengthens the duration of pulse checks, thereby adversely affecting CPR quality and outcomes. A number of studies have reported factors that prolonged the pause 
duration, such as defibrillation, changing of chest compression performers, and application of CPR device[16, 17]. To our knowledge, there is still no data exploring the impact of US on CCF.

The FEEL study demonstrated that US during CPR could be done within $10 \mathrm{~s}$ during pulse checks, compliant with the maximal pause duration of chest compressions[4]. By contrast, previous studies showed that US-related pause duration would be lengthened to 17-21s[15, 18-20]. However, this could be shortened by the implementation of a structured US protocol and the presence of US-trained faculties[7, $20,21]$. Whether US impacts negatively on CCF remains unclear.

Moreover, US could be done during the chest compression hands-on period with justified imaging quality[22]. This raises the possibility that US could be continued from hands-off to hands-on period (continuous US), potentially minimizing the interruptions of CPR. To date, evidence regarding the effect of continuous US on pause duration of pulse checks is limited.

Therefore, we conducted a retrospective observational study to evaluate the effect of US on CCF during CPR through video analysis. The effect of continuous US on pause duration of pulse checks was also investigated.

\section{Materials And Methods}

\section{Study design and setting}

This retrospective observational study was conducted from April 2017 to May 2018 at the ED of National Taiwan University Hospital. The study was approved by the Institutional Review Board of the hospital and registered at the ClinicalTrials.gov (NCT03695536).

Consecutive adult patients (more than 20 years of age, according to the civil law regulations in Taiwan) with cardiac arrest at the ED were eligible. Patients with age younger than 20 years, preexisting do-notresuscitate (DNR) order, traumatic arrests and video recording with poor quality were first excluded. Patients with DNR signed by the family during CPR were also excluded. None of the subjects had been in any patient cohort from our previous study[7].

Senior emergency residents and an organized team were responsible for resuscitation of the patients with cardiac arrest at the resuscitation area. The senior emergency residents, serving as sonographers, all completed basic emergency US training (certified by the Taiwan Society of Emergency Medicine) and the resuscitative US training curriculum[21, 23]. All of them passed the immediate evaluation and the reevaluation 6 months later, and showed their US proficiency at the real resuscitative setting $[7,23]$. The sonographic technique of continuous US was introduced during the monthly US teaching round of the ED and incorporated into resuscitation scenarios.

A timer was regularly employed during resuscitation, alarming every 2 minutes to remind the CPR team for pulse-check or rhythm analysis, and $10 \mathrm{~s}$ thereafter for resumption of chest compressions. A US 
machine (Noblus, Hitachi, Japan) equipped with 2-5 MHz curvilinear transducers was kept ready for use.

Video cameras have been approved and settled in the resuscitation room for recording of the CPR process for quality review. Recorded videos were stored in a secure hospital database. They were downloaded onto an encrypted hard-drive and reviewed by two researchers separately, using a standardized extraction form. If disagreement occurred, a third member was consulted until consensus was achieved. The faces of senior emergency residents were masked and blinded to the reviewers.

The total resuscitation duration, total pause duration and manpower during resuscitation were recorded. CCF was calculated as followings:

\section{See Formula 1 in the Supplemental Files}

If intermittent CPR occurred, the pause duration would be recollected when starting the next CPR. The causes of pauses were also collected.

The pause duration for pulse checks was recorded with stratification into those with and without simultaneous US. Those with US were further stratified into US during only hands-off periods (hands-off US), and continuous US. The reviewers would determine a CPR pause related to pulse checks by the timer alarming.

Clinical data were collected, including age, gender, previous medical history, initial cardiac rhythm, US scanning targets, sonographic findings, cause of cardiac arrest, application of extracorporeal membrane oxygenation (ECMO) devices, and outcomes including ROSC, survival to admission and hospital discharge.

The patients were categorized into two groups: patients receiving US during CPR (CPR with US group) and those without US (CPR without US). The primary outcome was CCF in the CPR with and without US groups. The secondary outcome was pause duration of pulse checks, comparing hands-off US with continuous US.

\section{Statistical analysis}

All data were analyzed by SAS software (SAS 9.4, Cary, North Carolina, USA). Duration were presented with mean \pm standard deviation. Student t-test was employed for continuous data, while Chi-square test for categorical data. $P$ value of less than 0.05 was considered statistically significant.

\section{Results}

A total of 213 patients were collected from April 2017 to May 2018. After exclusion of the patients with traumatic cardiac arrests, do-not-resuscitative orders, poor video quality, 153 patients were included in the current analysis (Fig. 1). Of them, 116 patients received US during resuscitation. The remainder received ALS without introduction of US. ROSC was achieved in 60 patients (39\%) and $17(11 \%)$ survived to hospital discharge. 
In the CPR with US group, all of the patients received continuous US at least for one time. Although total resuscitation time and total pause duration were longer in the CPR with US group, CCF was similar with that in the CPR without US group, as well as rates of ROSC, survival to admission and survival to discharge (Table 1).

Pause duration of pulse checks was investigated. In the CPR with US group, pause duration was longer in US-related pulse checks than that without US $(9.3 \pm 8.0$ v.s. $7.3 \pm 4.7 \mathrm{~s}, p<0.0001)$ (Table 2$)$. Of them, longer pause occurred in the hands-off US, comparing with that in the continuous US $(18.3 \pm 16.4$ v.s. $7.7 \pm 3.5 \mathrm{~s}$, $\mathrm{p}<0.0001)$. For pulse-checks without US in the CPR with and without US groups, however, there was relatively shorter pause in the CPR with US group $(7.3 \pm 4.7$ v.s. $8.9 \pm 7.2 s, p=0.0009)$.

When further analyzing the US scanning targets in the CPR with US group, subxiphoid view of heart was the most frequently checked target during pulse-checks. (Table 3). Aorta to evaluate for the existence of abdominal aortic aneurysm or aortic dissection, and extended focused assessment of sonography for trauma (eFAST) to evaluate the existence of free peritoneal fluid, indicative of internal bleeding, were the following targets during chest compressions in continuous US.

When looking into the sonographic findings during CPR, pericardial effusion was detected in 6 patients. Although aortic dissection was confirmed in 3 of the 4 patients with cardiac tamponade, they died after resuscitation. One patient was diagnosed to have bloody ascites and a ruptured abdominal aortic aneurysm was highly suspected during chest compressions. In addition, 62 out of the 116 patients receiving US were detected sonographic cardiac activity during CPR. The chance to achieve ROSC was significantly higher in this group of patients ( $32 / 62$ v.s. $10 / 54, p=0.0002)$.

\section{Discussion}

Whether US delays resumption of chest compressions and impacts on CPR quality remain a great debate. The results in this study showed US did not impact on CCF. In addition, pause duration of pulse checks could be lessened by using continuous US. If the sonographer insisted or tried to finish US before the resumption of chest compressions, the pause duration was much longer. On the contrary, if he/she allowed resumption of chest compressions and continued US (continuous US), the pause could become much shorter.

In addition to a single interruption of chest compressions, an even more important indicator to monitor quality of $\mathrm{CPR}$ is $\mathrm{CCF}$, a fraction of chest compressions duration along the whole CPR process. The American College of Cardiology (ACC)/American Heart Association (AHA) consensus statement recommends a target CCF of more than $60 \%$ and at best $>80 \%[9,24]$. To date, most of the studies regarding resuscitation US reported merely the length of pause rather than $\operatorname{CCF}[15,18-20]$. Whether lengthening of a single pause would compromise CCF is still unclear. In the current study, the high CCF of $92 \%$ was in accordance with the recommendation of the guidelines. The introduction of US during CPR did not influence CCF and survival. The results imply that US during CPR does not prolong overall no-flow time and influence the survival, although US may prolong an individual pause. However, in the CPR with 
US group, pause became shorter in pulse-checks without US. It possibly compensated for longer USrelated pause. Therefore, a similar CCF was preserved, comparing with the CPR without US group.

Such a high CCF in this study may be explained by adequate manpower, structured ALS teamwork, monthly review with continued training, and employment of a timer. In the current study, at least 7 members involved in each resuscitation scenario. The sonographer, all had completed basic US and resuscitative US training[21]. Through continued practice and accumulation of experience, the sonographers exhibited excellent US proficiency[7, 21]. In addition, a pre-set timer is important, which reminds the resuscitation team keeping pause as short as possible. Without the timer, the sonographer would tend to focus on sonographic examination and overlook time elapsing. Moreover, a high-quality portable ultrasound machine ready at the resuscitation room is essential. This helps speed up imaging diagnosis and shortening the interruptions. All of them contribute to whole CPR process in an orchestrated way.

Previous studies reported the pause with US could be prolonged to $17-21 \mathrm{~s}[15,18,19]$. Clattenburg et al reported shorter pause could be achieved when US was performed by an experienced sonographer or implementation of a structured US protocol[19, 20]. Nevertheless, all these studies focused on US performance during each pulse-check. Evidence regarding CCF and survival were limited. The study for the first time demonstrated that CCF, would not be reduced by employment of US.

This study is characterized by testifying the feasibility of continuous US. The results showed that such breakthrough in concept and intervention would largely broaden the time window of US assessment during CPR. US could be feasible during chest compressions with minimal interruption of CPR.

When performing US during CPR, there are several important organs to target. Subxiphoid view of the heart was the most often scanning target in the hands-off period, to focus on cardiac activity, pericardial effusion, right ventricular size, and left ventricular regional wall motion abnormalities. In the hands-on period, aorta and eFAST were frequently checked. These results to some extent explain how chest compressions might interfere US visualization of certain targets, and how the sonographer would choose in different periods during CPR to search for possible reversible causes at the earlier time point. Subxiphoid view of the heart is more suitable during hands-off period, since it is very close to the location of compressions and the image quality should be better when compression is held. For other targets far from the site of chest compressions, US is feasible during chest compressions. In one case, bloody ascites related to ruptured abdominal aortic aneurysm was detected during compressions. Using such approach of continuous US, not only the pause of chest compressions could be shortened, the reversible cause could potentially be identified and would be managed earlier.

Despite of the contributions, there are several limitations in this study. First, the results were obtained from a single institution. The facilities in the resuscitation room may vary from hospital to hospital. However, the study provides a possible way to improve quality of resuscitation while integration of US into CPR process. Second, selection bias could exist because the results were collected through retrospective video analysis. How to select patients for US during CPR was unclear. However, relatively 
shorter resuscitation time was noted in the CPR without US group. It was possibly explained that lots of work should be done in the early period of real resuscitation condition, such as endotracheal intubation, defibrillation and application of CPR device. US would be performed after aforementioned chaotic conditions. Third, the quality of US images was not included for analysis. Better quality of focused cardiac US images was suggested to be obtained during rhythm analysis and bag-mask ventilations[25]. In this study, each patient received 1-2 times of US evaluation. Not all US images were stored each time, though the key images with important findings were recorded. Fourth, the correlation between the US findings and the decision making or following management is not analyzed. The impact of the US findings on survival is harder to interpret. For example, cardiac tamponade resulting from aortic dissection was confirmed in 3 patients. The finding of US evidence of cardiac tamponade is almost always followed by pericardiocentesis. However, even early detection, it can hardly reverse the dismal outcomes. The potential value of resuscitative US on survival would be compromised.

\section{Conclusions}

The introduction of US during CPR does not impact on CCF. The individual pause would not be lengthened if the sonographer perform continuous US while allowing chest compressions to be resumed. In addition, a structured US training, adequate facilities and manpower, and a mechanism reminding resumption of chest compressions (such as a timer) would help sophisticated integration of US into CPR process.

\section{Declarations}

Ethics approval and consent to participate: The study was approved by the Institutional Review Board of the National Taiwan University Hospital (201807084RINB) and registered at the ClinicalTrials.gov (NCT03695536).

Consent for publication: Informed consents were waived by the Institutional Review Board of the National Taiwan University Hospital.

Availability of data and materials: Data sharing is not applicable to this article as no datasets were generated during the current study.

Competing interests: The authors declare that they have no competing interests.

\section{Funding: none.}

Authors' contributions: WCL, MHM and WTC conceived the study, designed the trial, and obtained research funding. MHM, WTC and WJC supervised the conduct of the trial and data collection. WCL, KMC, $\mathrm{HCC}$, and SFC undertook recruitment of patients and managed the data, including quality control. WCL and WTC provided statistical advice on study design and analyzed the data; WCL and WTC chaired the 
data oversight committee. WCL drafted the manuscript, and all authors contributed substantially to its revision. WTC and MHM take responsibility for the paper as a whole.

Acknowledgements: Not applicable.

\section{Abbreviations}

Ultrasonography (US);

Emergency departments (EDs);

Chest compression fraction (CCF);

Advanced life support (ALS);

Cardiopulmonary resuscitation (CPR);

Return of spontaneous circulation (ROSC);

Do-not-resuscitate (DNR);

Extracorporeal membrane oxygenation (ECMO);

Extended focused assessment of sonography for trauma (eFAST);

American College of Cardiology (ACC)/American Heart Association (AHA).

\section{References}

1. Perera P, Mailhot T, Riley D, Mandavia D: The RUSH exam: Rapid Ultrasound in SHock in the evaluation of the critically III.Emerg Med Clin North Am 2010, 28(1):29-56.

2. Volpicelli G, Lamorte A, Tullio M, Cardinale L, Giraudo M, Stefanone V, Boero E, Nazerian P, Pozzi R, Frascisco MF: Point-of-care multiorgan ultrasonography for the evaluation of undifferentiated hypotension in the emergency department.Intensive Care Med 2013, 39(7):1290-1298.

3. Lichtenstein DA: BLUE-protocol and FALLS-protocol: two applications of lung ultrasound in the critically ill.Chest 2015, 147(6):1659-1670.

4. Breitkreutz R, Price S, Steiger HV, Seeger FH, Ilper H, Ackermann H, Rudolph M, Uddin S, Weigand MA, Müller $\mathrm{E}$ et al: Focused echocardiographic evaluation in life support and peri-resuscitation of emergency patients: a prospective trial.Resuscitation 2010, 81(11):1527-1533.

5. Breitkreutz R, Walcher F, Seeger FH: Focused echocardiographic evaluation in resuscitation management: concept of an advanced life support-conformed algorithm. Crit Care Med 2007, 35(5 Suppl):S150-161. 
6. Chou HC, Chong KM, Sim SS, Ma MH, Liu SH, Chen NC, Wu MC, Fu CM, Wang CH, Lee CC et al: Realtime tracheal ultrasonography for confirmation of endotracheal tube placement during cardiopulmonary resuscitation.Resuscitation 2013, 84(12):1708-1712.

7. Lien WC, Hsu SH, Chong KM, Sim SS, Wu MC, Chang WT, Fang CC, Ma MH, Chen SC, Chen WJ: USCAB protocol for ultrasonographic evaluation during cardiopulmonary resuscitation: validation and potential impact. Resuscitation 2018, 127:125-131.

8. Monsieurs K, Nolan J, Bossaert L, Greif R, Maconochie I, Nikolaou N, Perkins G, Soar J, Truhlář A, Wyllie J: European Resuscitation Council Guidelines for Resuscitation 2015: Section 1. Executive summary.Resuscitation 2015, 95:1-80.

9. Nolan J: High-quality cardiopulmonary resuscitation.Curr Opin Crit Care 2014, 20(3):227-233.

10. Berg RA, Sanders AB, Kern KB, Hilwig RW, Heidenreich JW, Porter ME, Ewy GA: Adverse hemodynamic effects of interrupting chest compressions for rescue breathing during cardiopulmonary resuscitation for ventricular fibrillation cardiac arrest. Circulation 2001, 104(20):2465-2470.

11. Sanders AB, Kern KB, Berg RA, Hilwig RW, Heidenrich J, Ewy GA: Survival and neurologic outcome after cardiopulmonary resuscitation with four different chest compression-ventilation ratios.Ann Emerg Med 2002, 40(6):553-562.

12. Christenson J, Andrusiek D, Everson-Stewart S, Kudenchuk P, Hostler D, Powell J, Callaway CW, Bishop D, Vaillancourt C, Davis D et al: Chest compression fraction determines survival in patients with out-of-hospital ventricular fibrillation.Circulation 2009, 120(13):1241-1247.

13. Vaillancourt C, Everson-Stewart S, Christenson J, Andrusiek D, Powell J, Nichol G, Cheskes S, Aufderheide TP, Berg R, Stiell IG et al: The impact of increased chest compression fraction on return of spontaneous circulation for out-of-hospital cardiac arrest patients not in ventricular fibrillation.Resuscitation 2011, 82(12):1501-1507.

14. Soar J, Nolan JP, Böttiger BW, Perkins GD, Lott C, Carli P, Pellis T, Sandroni C, Skrifvars MB, Smith GB et al: European Resuscitation Council Guidelines for Resuscitation 2015: Section 3. Adult advanced life support.Resuscitation 2015, 95:100-147.

15. Huis In 't Veld MA, Allison MG, Bostick DS, Fisher KR, Goloubeva OG, Witting MD, Winters ME: Ultrasound use during cardiopulmonary resuscitation is associated with delays in chest compressions. Resuscitation 2017, 119:95-98.

16. Sell RE, Sarno R, Lawrence B, Castillo EM, Fisher R, Brainard C, Dunford JV, Davis DP: Minimizing preand post-defibrillation pauses increases the likelihood of return of spontaneous circulation (ROSC).Resuscitation 2010, 81(7):822-825.

17. Souchtchenko SS, Benner JP, Allen JL, Brady WJ: A review of chest compression interruptions during out-of-hospital cardiac arrest and strategies for the future.J Emerg Med 2013, 45(3):458-466.

18. Reed MJ, Gibson L, Dewar A, Short S, Black P, Clegg GR: Introduction of paramedic led Echo in Life Support into the pre-hospital environment: The PUCA study.Resuscitation 2017, 112:65-69.

19. Clattenburg EJ, Wroe P, Brown S, Gardner K, Losonczy L, Singh A, Nagdev A: Point-of-care ultrasound use in patients with cardiac arrest is associated prolonged cardiopulmonary resuscitation pauses: $A$ 
prospective cohort study.Resuscitation 2018, 122(1):65-68.

20. Clattenburg EJ, Wroe PC, Gardner K, Schultz C, Gelber J, Singh A, Nagdev A: Implementation of the Cardiac Arrest Sonographic Assessment (CASA) protocol for patients with cardiac arrest is associated with shorter CPR pulse checks. Resuscitation 2018, 131:69-73.

21. Lien WC, Hsu SH, Chong KM, Sim SS, Wu MC, Chang WT, Fang CC, Ma MW, Chen SC, Chen WJ: Data on evaluation of proficiency for the US-CAB curriculum.Data in Brief 2017, 17:965-968.

22. Lien WC, Chang WT, Ma MH, Chen WJ: Ultrasonography during CPR: Hands-off or hands-on? Resuscitation 2018, 125:e8-e10.

23. Lien WC, Liu YP, Chong KM, Sim SS, Wu SH, Wu CY, Wu MC, Chang WT: A novel US-CAB protocol for ultrasonographic evaluation during cardiopulmonary resuscitation.Resuscitation 2017, 115:e1-e2.

24. Meaney PA, Bobrow BJ, Mancini ME, Christenson J, de Caen AR, Bhanji F, Abella BS, Kleinman ME, Edelson DP, Berg RA et al: Cardiopulmonary resuscitation quality: improving cardiac resuscitation outcomes both inside and outside the hospital: a consensus statement from the American Heart Association.Circulation 2013, 128(4):417-435.

25. Aagaard R, Løfgren B, Grøfte T, Sloth E, Nielsen RR, Frederiksen CA, Granfeldt A, Bøtker MT: Timing of focused cardiac ultrasound during advanced life support - A prospective clinical study.Resuscitation 2018, 124:126-131.

\section{Tables}


e 1. The demographic data of the included patients.

\begin{tabular}{|c|c|c|c|c|}
\hline racteristics & $\begin{array}{c}\text { Total } \\
(\mathrm{n}=153)\end{array}$ & $\begin{array}{l}\text { CPR with US* } \\
\quad(n=116)\end{array}$ & $\begin{array}{l}\text { CPR without US } \\
(\mathrm{n}=37)\end{array}$ & $p$ Value $^{\dagger}$ \\
\hline$\overline{(\text { years) }})^{\ddagger}$ & $69.2 \pm 16.6$ & $69.0 \pm 16.6$ & $70.2 \pm 16.8$ & \\
\hline gender, $n(\%)$ & $93(61 \%)$ & $72(62 \%)$ & $21(57 \%)$ & \\
\hline \multicolumn{5}{|l|}{ ical history, $n(\%)$} \\
\hline abetes mellitus & $43(28 \%)$ & $31(30 \%)$ & $7(20 \%)$ & \\
\hline pertension & $82(54 \%)$ & $64(55 \%)$ & $18(48 \%)$ & \\
\hline rdiac diseases $^{\S}$ & $59(39 \%)$ & $44(38 \%)$ & $15(40 \%)$ & \\
\hline lmonary diseases ${ }^{\S}$ & $11(7 \%)$ & $8(7 \%)$ & $3(8 \%)$ & \\
\hline nal diseases ${ }^{\S}$ & $28(19 \%)$ & $21(18 \%)$ & $7(20 \%)$ & \\
\hline alignancy & $25(16 \%)$ & $16(14 \%)$ & $9(24 \%)$ & \\
\hline rebrovascular accident & $22(14 \%)$ & $15(13 \%)$ & $7(20 \%)$ & \\
\hline al shockable rhythm, $n(\%)$ & $18(12 \%)$ & $15(13 \%)$ & $3(8 \%)$ & \\
\hline \multicolumn{5}{|l|}{ se of cardiac arrest, $n(\%)$} \\
\hline rdiogenic & $78(51 \%)$ & $63(54 \%)$ & $15(41 \%)$ & \\
\hline way & $31(20 \%)$ & $22(19 \%)$ & $9(24 \%)$ & \\
\hline hers & $44(28 \%)$ & $31(27 \%)$ & $13(35 \%)$ & \\
\hline \multicolumn{5}{|l|}{ power during resuscitation, $n^{\ddagger}$} \\
\hline nior physician & $1.8 \pm 0.7$ & $1.8 \pm 0.7$ & $1.7 \pm 0.5$ & \\
\hline aior physician & $0.9 \pm 0.9$ & $0.9 \pm 0.9$ & $0.8 \pm 1.0$ & \\
\hline Irse & $5.9 \pm 1.8$ & $5.9 \pm 1.8$ & $5.6 \pm 1.7$ & \\
\hline ication of ECMO* devices, $n(\%)$ & $16(10 \%)$ & $13(11 \%)$ & $3(8 \%)$ & \\
\hline 1 resuscitation duration $(\mathrm{s})^{\ddagger}$ & $1441.8 \pm 720.6$ & $1543.0 \pm 687.6$ & $1024.7 \pm 716.5$ & 0.0011 \\
\hline l pause duration $(\mathrm{s})^{\ddagger}$ & $99.6 \pm 57.8$ & $108.2 \pm 58.5$ & $63.6 \pm 37.9$ & 0.0004 \\
\hline st compression fraction $(\%)^{\ddagger}$ & $92.6 \pm 3.3$ & $92.6 \pm 3.2$ & $92.6 \pm 3.9$ & \\
\hline Irn of spontaneous circulation, $n(\%)$ & $60(39 \%)$ & $42(36 \%)$ & $18(48 \%)$ & \\
\hline ival to hospital admission, $n$ (\%) & $58(38 \%)$ & $42(36 \%)$ & $16(44 \%)$ & \\
\hline ival to discharge, $n(\%)$ & $17(11 \%)$ & $13(11 \%)$ & $4(12 \%)$ & \\
\hline
\end{tabular}

R, cardiopulmonary resuscitation; US, ultrasonography; ECMO, extracorporeal membrane oxygenation. nparisons between CPR with and without US groups.

ressed as mean \pm standard deviation.

diac diseases included coronary artery disease, heart failure and arrhythmia; pulmonary diseases included nchial asthma and chronic obstructive pulmonary disease; renal diseases included chronic renal insufficiency, l end-stage renal disease receiving dialysis. 
Table 2. Pause duration of pulse checks.

\begin{tabular}{lcc}
\hline Type of pulse check & $\mathrm{n}$ Pause duration (s) \\
\hline CPR with US* group & & \\
\hline Pulse checks with US & 150 & $9.3 \pm 8.0^{\dagger}$ \\
\hline Continuous US & 127 & $7.7 \pm 3.5^{\S}$ \\
\hline Hands-off US & 23 & $18.3 \pm 16.4^{\S}$ \\
\hline Pulse checks without US878 & $7.3 \pm 4.7^{\dagger \ddagger}$ \\
CPR without US group & \\
\hline Pulse checks without US 186 & $8.9 \pm 7.2^{\ddagger}$ \\
\hline
\end{tabular}

*CPR, cardiopulmonary resuscitation; US, ultrasonography.

$\dagger_{\mathrm{p}}<0.0001$

${ }_{\mathrm{p}} \mathrm{p}=0.0009$

$\S_{\mathrm{p}}<0.0001$

Table 3. Ultrasonographic evaluation in the CPR with US* group.

\begin{tabular}{lcc}
\hline Scanning targets & $\begin{array}{c}\text { Hands-off USContinuous US } \\
(\mathrm{n}=27)\end{array}$ & $(\mathrm{n}=123)$ \\
\hline Heart & 27 & 44 \\
Heart-aorta & - & 41 \\
\hline Heart-aorta-eFAST & - & 38 \\
\hline
\end{tabular}

*CPR, cardiopulmonary resuscitation; US, ultrasonography; eFAST, extended focused assessment of sonography for trauma.

\section{Figures}

\section{Figure 2}

The study flow diagram. CPR, cardiopulmonary resuscitation; US, ultrasonography; ROSC, return of spontaneous circulation. 